\title{
Sulhalţ̌uerzeiduni
}

\section{(Erftes fud.}

\section{Ctatwejen}

Geite

I. Berfaffungaredttliche (Srundlage und Bebeutung bes Staatahaus= balt $3=$ Crtat?

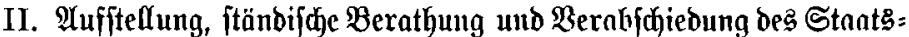
hau 3 halt $\bar{s}=$ Etats .

III. Formelle Cinridytung bes Stantshaushalts=@tats

IV. Statrechtliche Brundjäbe in Bezug auf bie Bebahrung mit ben

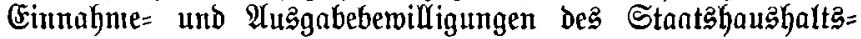
Etate

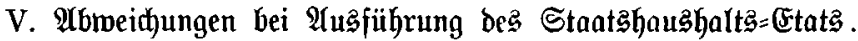

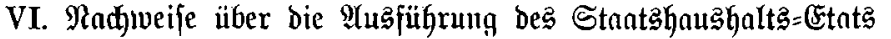

Reilagen A-F

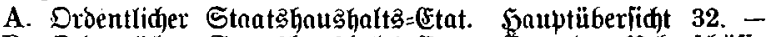

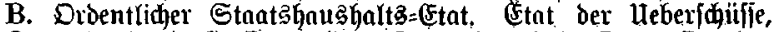
Fap. 1144 - C. Drdentlider Staatshau3halt =E tat. Etat ber

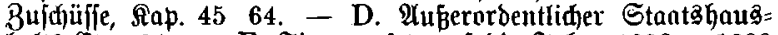
halt's= Etat 84. - E. Finanzgeles auf bie Jahre 1882 น. 1883

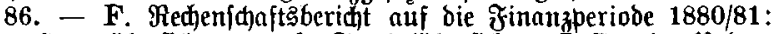
a. Souptiiberfidt 88. - b. Spezialüberfigten: I. Etat ber Ueber=

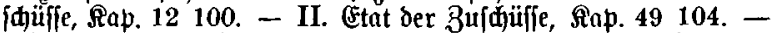
c. Meberfidt ber auperordentliden Einnabmen und $\mathfrak{A}$ agaben 110.

\section{Bweites Bud.}

\section{Maffentwejen}

I. Spezialfaffen und Bentralfaffen bes Stantahaußhalts . . 116

II. Buafüührung bei ben $\Re$ affen . . . . . . . . . . . 141

III. 3uläffige Zahlungsmittel im Berfehre ber ftnatlichen Raffen .

IV. Bebandlung ber bei Stantafaffen eingebenben nachgemaudten unt berfäriduten formie befdäbigten und unbraudbar getworbenen

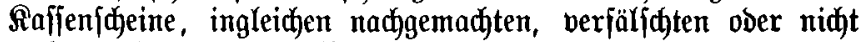
mehr umlaufşähigen Münzen

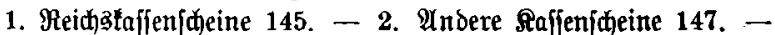

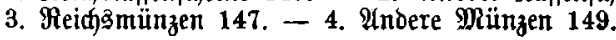


V. Berinahrung und Bertretumg ocr Aafjenteftünde.

VI. Berpaffung ber Siaffengelder . . . . . . . . . . . 161

I. Borjdriften für bas Berpađen der Reidşmünzen 161. -

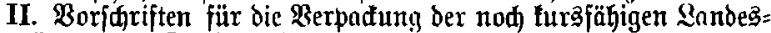
münzen der Thalerwährung 163.

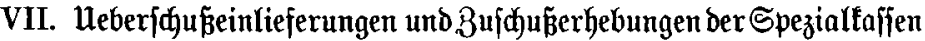

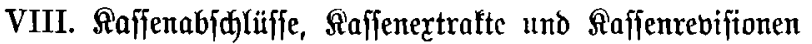

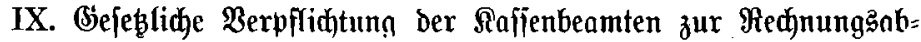
legung

X. Dienfffautionen ber Saffenbeamten.

\section{Drittes Budd.}

Hechnungsiwejen

Grffer Offeif. $\mathfrak{A}$ Ilgemeine $\mathfrak{B o r f d x r i f t e n ~ f u ̈ r ~ b a s ~ S t a a t s = ~}$ red)nungatwefen

Eriter Ahidnitt. Arrgemcine (Brundfäßze für bic Berred)= nung ber Einnahmen und 2 usgaben de马 Staates.

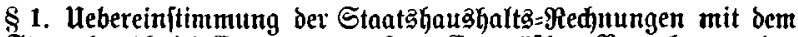

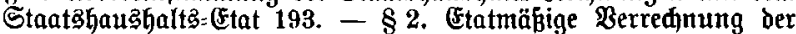

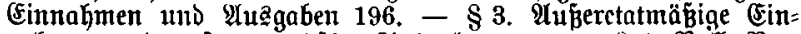

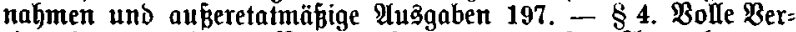

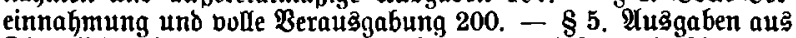

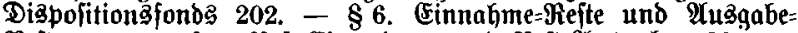
Refte 205. - \$ 7. Reit=-Einnahmen uno Reit=2lusgaben 207. \$8. Bü̆therabiallus bei ben Spezialfaffen 207. - \$9. Büber=

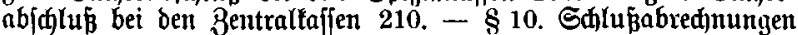
Der Spezialtafien mit ber betreffenden Zentraltaffe 212 . - \$11.

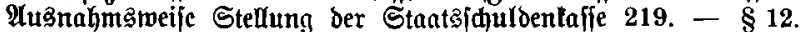

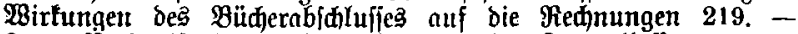

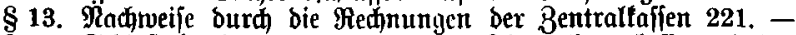

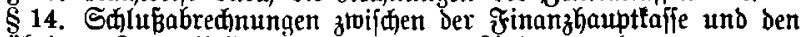
übrigen Bentralfaffen 222, - \& 15 . Ünhangarednung $z^{4}$ ben Strat:3hau 227. - \$ 17. Bseneral=Stantahaushalt

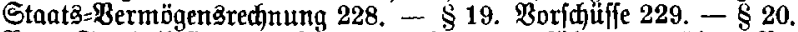
$\mathfrak{B o n}$ Speziallaffen an bejondere Ređfnungäübrer gewäbrte $\mathfrak{B e}=$ rechnungeggelder 233. - \$21. Eiferme Beftände der Speziallaffen 234. $-\$ 22$. Berivahrurgspojten 234. $-\$ 23$. Aqusgabe=\{ejer= vate 235, - \$24. Eriparnifie an ben Etatjummen 236.

Bweiter Mbfdnitt. Urgemeine Borfdriften über bie Ein= rid) tung ber $\Re$ ednungen.

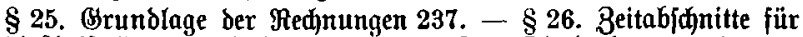

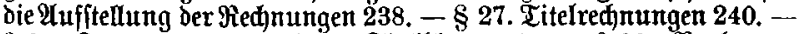
\$28. Format 241. $-\$ 29$. Titelblatt 242. $-\$ 30$. Borbemer= lungen 243. $-\$ 31$. Reinffifrift 244.. - $\$ 32$. Beridtigungen ber

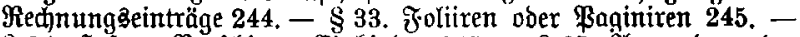
\$34. Sheften, Broifhiren, Einfinden 245. - \$35. Üntwentoung ber

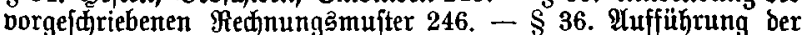
einzelnen (Etattitel 246. $-\S 37$. Rontext 247. $-\$ 38$. Erläute=

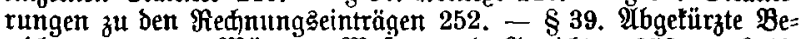
zeidinungen von Münzen, Maken uno Brewidten 252 . - $\$ 40$. Berlautbarung der Belegnummern 255. $-\$ 41$. $\mathfrak{A b}$ [d Belbređ̆nungen 256. - \$42. Boŭzziełung 258. 
Dritter Abjunitt. Borfdriften über Die rednungamäßige

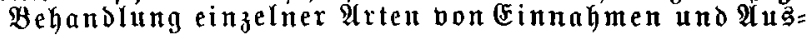
gaben.

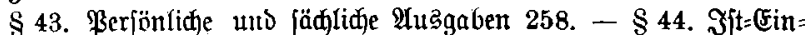

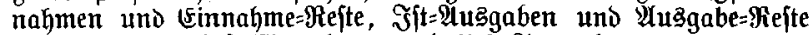

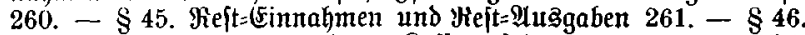

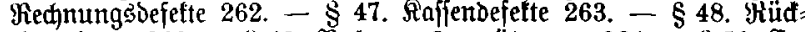
eimnahmen 263 . $-\$ 49$. Redmungäbergütungen 264 . $-\$ 50$. ( $\mathrm{r}=$ ftattungen 265. - $\$ 51$. Regełmäfig twieberfehrende bertrags $=$ mäbige Zahlungen 266. - \$52. Durdlaufende \$often 266. -

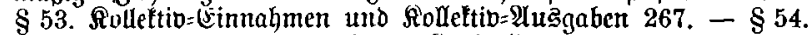

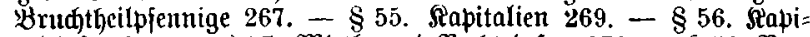
talzinfen 270. - \$ 57. Mieth= und \$adtzinfen 272.-\$58. Ber $=$ ausgabungen fïr Saturalien, Miaterialien uns Fabritate 273 . $\$ 59$. Dienfthezüuge 273. $-\$ 60$. Tagegelder und Yieifeloften 278 . - $\$ 61$. Qügaben für Sianzlei= oder Bürcaubedürfniffe 278. $\$ 62$. Werau@gabungen für J̈nnentariengegenjtände 279 . $-\$ 63$. *3oftporto, Telegraphengebühten, Eifenbahnfradten 280. - \$ 64 .

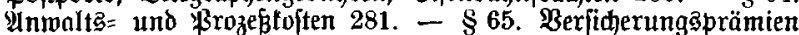

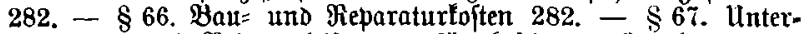
ftübungent uno Beitragsleiftungen für bejtimmte Brvecfe 283 . \$ 68. Jnventariengegenftände un๖ Sammlungaggegenftände 284. -

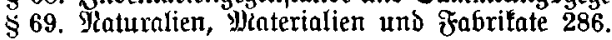

Bierter Mbiđnitt. Borjđriften über die Rednungăbelege.

I. Argemeine Borjariften.

\$70. Ulmfang der Belegpflidt 287. - \$71. Formelle Erforber niffic 289. - \$ 72. Signatur 295. - \$73. Beifeinigungen be

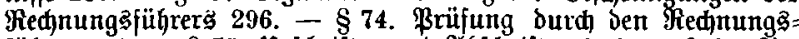

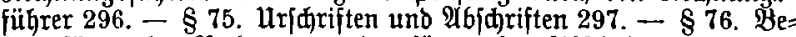

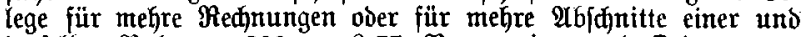
berjelben Mednung 299. - \$77. Mummeriren uns Dronen 299.

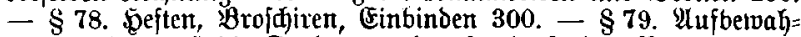

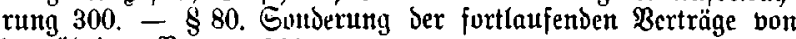
ben übrigen Belegen 301.

II. Borfdriften über einzelne Arten bon Belegen.

\$ 81. Raffentanmeijungen 302. - $\$ 82$. Berträge $302 .-\$ 83$. $\mathfrak{i} i z i=$

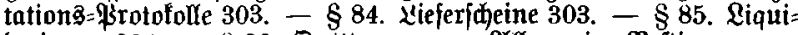
Dationen 304. - \$ 86. Duttungen. - Angemeine Béptimmungen

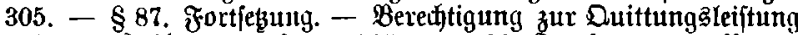
uno zum 3 aljlungaempfange 305. $-\lessgtr 88$. Fortjekung. - $\mathfrak{u}$ ter $=$ fdrift 308. - \$ 89. Fortfegung. - Dutttungen in Form von $\mathbb{Z a h}=$

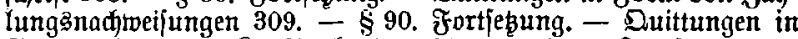

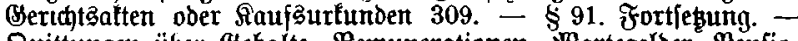
Dutitumgen iiber Behalte, Remunerationen, Wartegelder, Renfio= nen, fortlaufende Unterftügungen 310 . $-\$ 92$. Fortfęurg. Duttungen über 2Trbeitälöhne 311. - \$93. \&ortiegung. - Jahres= quittungen 314. - $\$$ 94. Fortietzung. — Suittungen bon Beifionaren und Erben 314. - \$ 95. Fortiegung. - Duttungen bon Ehe= frauen 315. - \$96. Fortfeßung. - Duittungen von Bormün= Derm 315. - \$97. Fortfekung. - Sluittungen iffentlidjer $\mathfrak{B} e=$ hörben 315. - \$ 98. F゙ortfeburtg. - Duttungen über Bahlungen

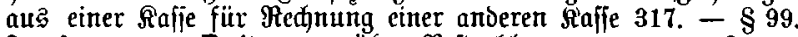

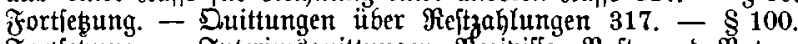

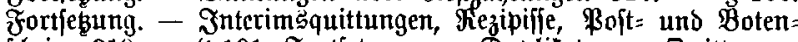
fateine 318. - \$101. Fortjekung. - Duplifate von Duttungen

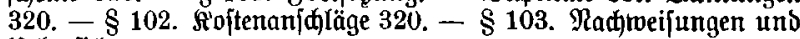
Ueberjidjten 320.

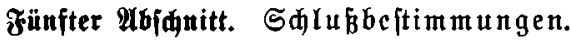

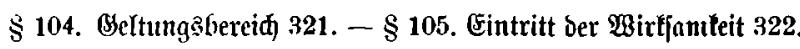




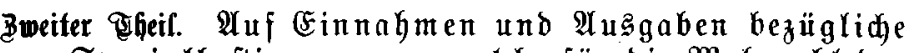
Spezialbeftimmungen, welde für bie Mefrzahl ber ftantliden Bebörben und saffen von praftifder gebeutung jind

seite

I. Dienitgenǘ der aftiben Staatabeamten und anderer öffentlidger Funftionäre . . . . . . . . . . .

II. Wartegeld quieझzirter Staatß̧beamter und anderer öffentlicher Junftionäre . . . . . . . . . . . .

III. Bellion verabjujiedeter Stantabeamter und anderer öffentlicher Funttionäre

IV. Bnadengenuf und ßenfion ber Sinterlaffenen von Staat3beamten und anderen öffentlicthen Funttionären

V. Beitragaleiftungen ber Staatabeamten und anderer offentlicher Funttionäre jür ßenjionzztwerte

VI. Umzugafoften bei Berfebung bon Staatabeamten und anderen iffentlichen Funftionären

VII. Tagegelder und Reifefojten bei Dienjitreijen von Stants= beamten umb anderen ïffentliden Funttionären

A. Tagegelder und Fteijefoiten bei Dienftreijen oon Staat 3beamten 421. - B. Iagegelder uno Reifeloften anderer öffentlidjer Funttionäre 454. -

VIII. ßojtporto und Eijenbahniradjten

IX. Telegraphengebuigren

X. Injertiongłoften . . . . . . . . . . . . . . . . . 501

XI. Branbverfidjerungafoften . . . . . . . . . 504

XII. Sgotbautoften . . . . . . . . . . . . 508

A. Baurebifionen und wauanjaläge 508. - B. Wau=

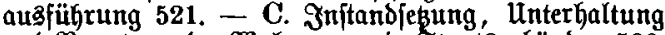
und Benubung ber Bohnungen in Staatæagebäuben 538 . - D. Bauredinungen 557.

XIII. Stempelabgaben von Mrfunden

XIV. Sreditixung (Seftundung) non Forderungen der Staats= tajfen.

XV. Erftattung bon 3ahlungen an bie Stantałaffen . $\quad 593$

XVI. (Erlaß̧ bon Forberungen der Staatßfaffen .

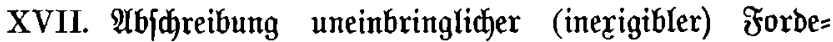
rungen der Stnatzlafjen.

XVIII. Berjährung Der Forderungen und Leiftungen ber Staatataffen und amberer ftaatlidjer Snjtitute

XIX. YYbtretung und Bfändung von (Gebalten, $\mathfrak{B a r t e g e l d e r n , ~}$ ßenftonen, Bnadengenú und c̈hnlidgen \&eiftungen Dex Staatä́affen

XX. ßmangsmeife Beitreibung bon (Seldleiftungen in $\mathfrak{B e r}=$ waltungsfactyen 


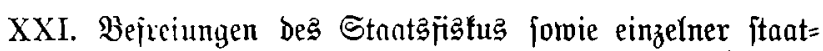
lid)er 5 rgane und $\mathfrak{Q}$ (nitalten von iffientlidjen Leiftungen und fonjtige finanzielle Redytsbergïnjtigungen Derfelben

XXII. Bertretung Des Stantäfizłt in redttlichen $\mathfrak{A}$ nelegen= geiten und Bieridtaftand befjelben.... . . . 668

\section{Wierteg Buh.}

\section{Staatahauß̧haltątontrole}

I. Brefuxidytliche Sntmidelung Der Staatahaushaltatontrole in sadffen .

II. Die Dberrednungätammer in ifren gegentärtigen (Finrid)= tungen und Befugnilien.

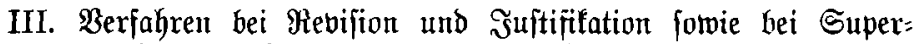
rebifion ber Re(d)mungen bur(f) bie Dberred)

1. Shegenitände, auf meldhe fïh bie Yiebifinn, beziehentlidh bie

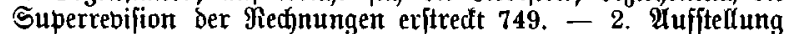
und Erlesigung ber (Erinnerungen ber Dberrednung ștammer 751.

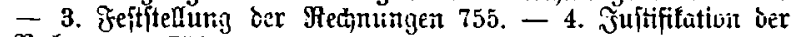
Redinumẹen 756. 


\section{(Errklärung Der hauptfüdlidłften Abkürłuntaen.}

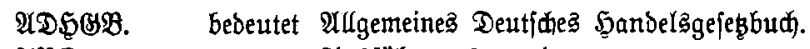

IIBD.

$\mathfrak{B} \mathfrak{B}$.

" $\mathfrak{A}$ ฉ̊ührungąberoromung.

() 38 .

$\mathfrak{F M}$.

(s).

BD.

BM.

BBP.

BBD.

IM.

JMPB.

$\Re \mathfrak{R}$.

M. ธ. Э.

DRR.

$\mathfrak{R H B}$.

BD.

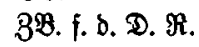

$3 \mathfrak{B}$.

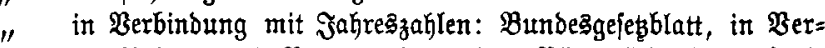

bindung mit Baragraphenzahlen: Bürgerlidjes (Gejeghud.

3:B.

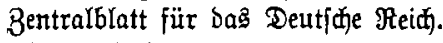

" ₹inangminifterium.

$"$ Beję.

" Seneralbireftion ber Stantäeifenbahnen.

" Bejammtminifterium.

"Bejes= und Beroronungäblatt.

"1 Beneralberorbnung.

" Э઼uโtizminifterium.

" Эultiz=-Minifterial=Blatt.

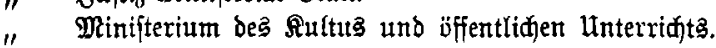

" Minifterium bes Innern.

" Eberredinungalammer.

" ReiđjagejeşGlatt.

" Berorbnutng.

" Boll= und Steuer=Direftion.

" $\quad$ Boll= und Steuer= Berorbnungeglatt. 